\title{
Small dams for aquaculture negatively impact fish diversity in Amazonian streams
}

\author{
Raniere Garcez Costa Sousa ${ }^{1, *}$, Marcos de Almeida Mereles ${ }^{1}$, \\ Flávia Kelly Siqueira-Souza ${ }^{2}$, Lawrence Edward Hurd ${ }^{3}$, \\ Carlos Edwar de Carvalho Freitas ${ }^{2}$
}

\begin{abstract}
${ }^{1}$ Fisheries Science Department, Rondônia Federal University, Presidente Médici, CEP 76916-000, Rondônia, Brazil ${ }^{2}$ Fisheries Science Department, Federal University of Amazonas, Manaus, CEP 69077-000, Amazonas, Brazil ${ }^{3}$ Department of Biology. Washington and Lee University, Lexington, VA 24450, USA
\end{abstract}

\begin{abstract}
Much has been written about the negative impacts of large hydroelectric dams on fish species diversity in the Amazon River Basin; however, less is known about the impacts of small dams in streams that are created for fish aquaculture. Our study of fish assemblages upstream and downstream of fish farm dams in Rondônia State, Brazil, revealed that the dams act as physical barriers to fish movement and that upstream assemblages showed lower measures of diversity and abundance compared to downstream. The greatest impact was the obstruction of upstream movement of a number of fish groups, coupled with isolation and disappearance of relatively rare fish species living upstream. The fish species most affected were from frugivore, herbivore and detritivore trophic levels that are associated with migration and the forming of schools (potamodromous species), although the impact was also evident in piscivorous fish commonly found in lentic habitats. Although stream dams may cause small negative effects relative to huge hydroelectric barriers, the cumulative impact of hundreds of fish farms in stream channels could be considerable. Amelioration of the damage caused by fish farm impoundments will require (1) design of effective fish passage systems around dams to reduce impact on fish diversity and (2) prohibition of the complete stream blocking to build these fish farms, which will require derivative channels to their water supply.
\end{abstract}

KEY WORDS: Fish assemblages $\cdot$ Dams $\cdot$ Stream basin $\cdot$ Tributaries

\section{INTRODUCTION}

There are $>154$ large hydroelectric dams in operation in the Brazilian Amazon, with 21 dams under construction and 277 more planned over the next few decades (Finer \& Jenkins 2012, Castello \& Macedo 2015) in order to supply the Brazilian demand for power (Agostinho et al. 2008). It is well known that these obstructions greatly alter the landscape and negatively affect aquatic ecosystems, including fragmentation and isolation of fish communities (Girard 2002, Khan et al. 2014, Hurd et al. 2016, Pereira et al. 2016, Winemiller et al. 2016). Dams interrupt the tim-

\footnotetext{
*Corresponding author: ranieregarcez@unir.br
}

ing and intensity of the annual hydrological cycle in the Amazon River Basin, which largely determines important life cycle characteristics of native fish such as dispersal during breeding seasons (Maltchik \& Medeiros 2006) and movement related to feeding opportunities (Hahn \& Fugi 2007). In particular, dams prevent migratory fish species from using river channels for reproductive movements (Ribeiro et al. 1995, Cox-Fernandes 1997, Freitas \& Garcez 2004, Sousa \& Freitas 2008, Miranda 2012). Fish ladders in this region have proven to be either ineffective or detrimental to fish survival (Pelicice \& Agostinho 2008, Agostinho et al. 2012, Pelicice et al. 2015). Both

() The authors 2018. Open Access under Creative Commons by Attribution Licence. Use, distribution and reproduction are unrestricted. Authors and original publication must be credited. 
upstream and downstream dispersal remains disrupted, and mortality of fish that are able to traverse the fish ladders tends to be high (Pelicice et al. 2015).

Although most research to date has focused on the impacts caused by large-scale hydroelectric barriers in major rivers (Winemiller et al. 2016), there are also many smaller dams built in streams for fish aquaculture (Neto et al. 2015, Lima et al. 2016). The impact of individual dams would likely be small, but the cumulative negative effects from multiple impoundments over an entire sub-basin could cause irreversible damage to the aquatic environment (Albanez \& Albanez 2000) and specifically to fish assemblages (Agostinho et al. 2007). To determine the number of aquaculture farms that a given watershed can support, it is essential to verify the impacts associated with the construction of small dams for fish farm impoundments (Agostinho et al. 2007). Apparently, almost all of the fish farms in Brazil already in operation are not up to code and have contributed to deforestation of riparian areas, fragmentation of river habitats, blockage of migratory routes and degradation of nursery areas (Barletta et al. 2010).

Rondônia State is located in the southern part of the Brazilian Amazon and currently has $>4000$ licensed fishing farms, mainly in the Machado River basin (Ostrensky et al. 2008, Rondônia 2015). These farms specialize in the production of tambaqui Colossoma macropomum (Cuvier 1818), a large-sized characiform species that is endemic to the Amazon basin (MPA 2010). Studies of the effects of aquaculture on native fish assemblages are very scarce in Amazonia and completely nonexistent in the Machado River basin. The present research aimed to identify the impacts of fish aquaculture farms on the wild fish fauna of the streams in the headwaters of the Machado River by testing the following null hypotheses: (1) that small aquaculture dams have no effect on the structure of fish communities upstream or downstream; and (2) that there are no environmental changes in water quality associated with these fish farms.

\section{MATERIALS AND METHODS}

\section{Study area}

The study area was located in the Machado River basin (Rondônia State, Brazil), where 5 streams were dammed to impound water for aquaculture farms (Fig. 1). All of the studied streams are shallow, loworder tributaries of the Machado River with sandy and rocky substrates. Grasslands are typically found on the margins of these streams, as well as some stretches of preserved primary and secondary forests, including in the riparian zone. The status of the selected environmental variables was assessed in the field, using notes and a metric rule. The aquaculture impoundments ranged in size from 1.03 to 17.14 ha (10300 to $171400 \mathrm{~m}^{2}$, Table 1), with no treatment systems in place to purify water before returning it to the stream. These aquaculture impoundments allowed us to investigate the effects of semi-intensive fish production since it is a system that directly interacts with the stream's main channel.

\section{Sample collections}

Fish were sampled in 2 consecutive years, during 2 periods of the annual hydrological cycle: low water (September 2014 and June 2015) and rising water (December 2014 and March 2015). Fish sampling was performed in $150 \mathrm{~m}$ long-sections upstream and downstream of fish farming impoundments at 5 small streams, giving a total of 10 sampling locations (1 upstream and 1 downstream of each fish farm). Fish were captured using 3 different types of apparatus that were employed at every sampling site: (1) 2 gill nets $2 \mathrm{~m}$ high and $5 \mathrm{~m}$ long with $20 \mathrm{~mm}$ mesh, applied transversally or on the border of the river channel; (2) 1 ring net trap with a diameter of $2 \mathrm{~m}$ and $10 \mathrm{~mm}$ mesh size between opposite knots, moving continuously for $2 \mathrm{~h}$, with 15 min intervals within long-sections (from one end to the other) of the stream; (3) 1 dip net with $2 \mathrm{~mm}$ mesh and $1 \mathrm{~m}$ diameter, also employed at the streams margins for a period of $2 \mathrm{~h}$. Both trap types were set for a period of $2 \mathrm{~h}$ at night (18:00 to 20:00 h) and during the day (06:00 to 08:00 h) and were inspected every $20 \mathrm{~min}$. At the same time, physical-chemical parameters were measured, including water temperature, dissolved oxygen, $\mathrm{pH}$ and electrical conductivity. The sampled fish were euthanized in cold shock (ice-water solution), labeled, stored in plastic bags and saved in an isothermal box for posterior taxonomic identification in the Aquaculture and Fisheries Laboratory at Rondônia Federal University.

\section{Data analysis}

Fish community composition values were initially subjected to descriptive analysis to determine frequencies, means and standard deviations. The following ecological parameters were also calculated: 


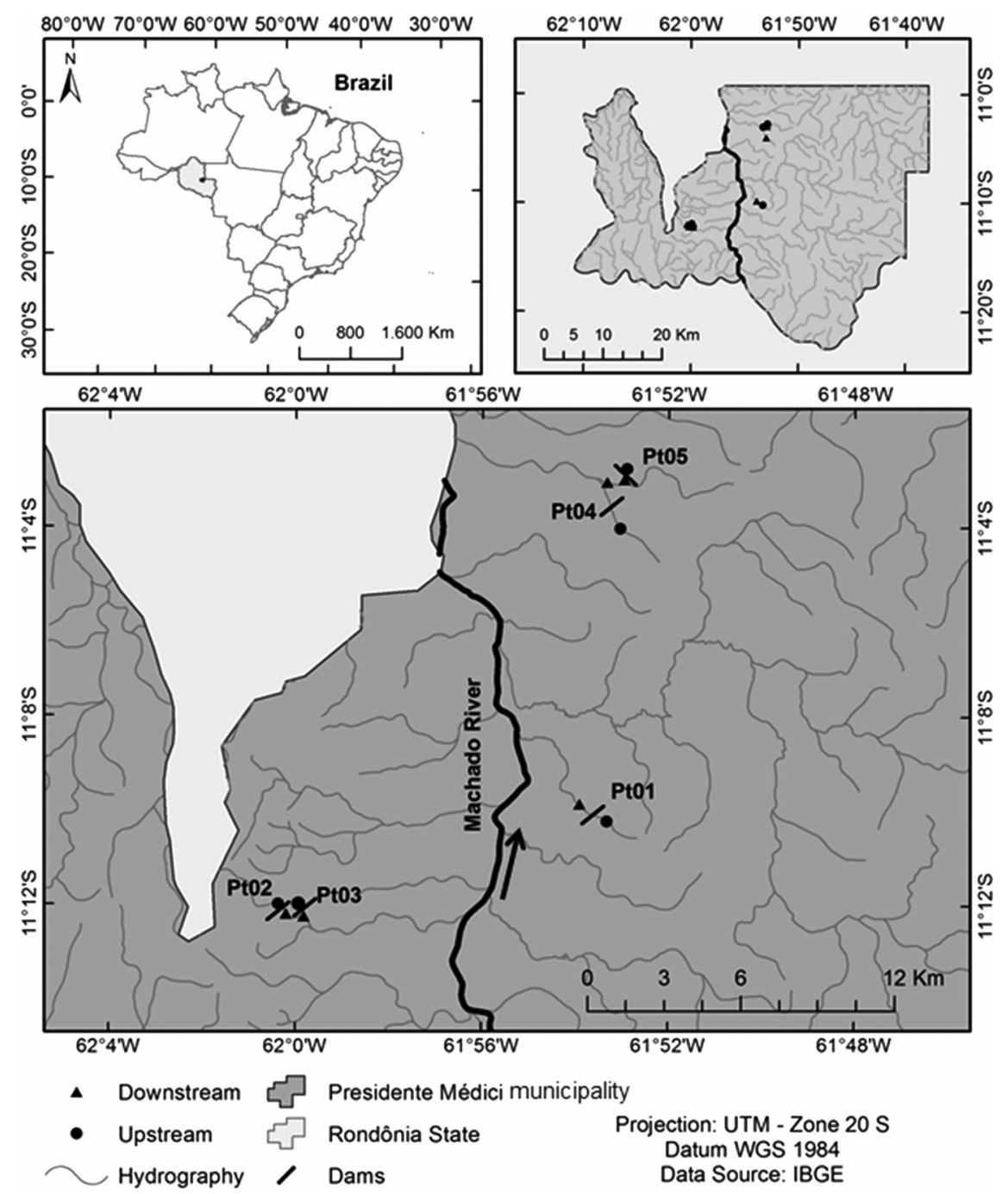

Fig. 1. Sampling sites and aquaculture dam locations in headwater streams of the Machado River basin, Rondônia State, Brazil. Arrow indicates the direction of flow of the Machado River

species richness $(S)$, Shannon's index of diversity $\left(H^{\prime}\right)$, Berger-Parker index of dominance $(D)$ and Evenness $(E)$ for both upstream and downstream data sets. Differences in water quality measurements between upstream and downstream data were tested with Student's $t$-test, when the data met the assumptions for normality and homoscedasticity. To test the null hypotheses that fish species composition is similar between upstream and downstream samples and between the low and high water seasons, a 2-way PERMANOVA was employed with 5000 permutations (Anderson 2001), based on a matrix of Canberra distance measures. The assumption of multivariate homogeneity of groups was tested before performing PERMANOVA. The Canberra distance was used to estimate the distance between sampling sites because this metric excludes double zeros and increases the effect of differences between variables with low values and many zeros (Buttigieg \& Ramette 2014).

We classified fish species according to 3 life history strategies adapted from Winemiller \& Rose (1992) and Winemiller (2005) based on their bioecologic characteristics (following Carvalho \& Tejerina-Garro 2015, Röpke et al. 2017 and Arantes et al. 2017): (1) equilibrium strategists: species with moderate to long generation time, low reproductive effort, variable body size, low batch fecundity, high investment per offspring and, in general, no migratory behavior (24 species); (2) periodic strategists: species with long generation time, moderate reproductive effort, medium to large body size, high batch fecundity, low investment per offspring and migratory behavior (14 species); and (3) opportunistic strategists: species with short generation 
Table 1. Environmental characteristics of sampling sites (Pt01-05, see Fig. 1). Mean \pm SD

\begin{tabular}{|c|c|c|c|c|c|}
\hline Parameter & Pt01 & Pt02 & Pt03 & Pt04 & Pt05 \\
\hline $\begin{array}{l}\text { Downstream average } \\
\text { channel width (m) }\end{array}$ & $2.75 \pm 0.65$ & $1.78 \pm 1.28$ & $1.20 \pm 0.81$ & $1.99 \pm 0.27$ & $1.09 \pm 0.14$ \\
\hline $\begin{array}{l}\text { Upstream average } \\
\text { channel width (m) }\end{array}$ & $2.01 \pm 0.30$ & $2.45 \pm 0.37$ & $2.25 \pm 0.78$ & $4.73 \pm 1.01$ & $1.09 \pm 0.14$ \\
\hline $\begin{array}{l}\text { Downstream average } \\
\text { depth }(\mathrm{m})\end{array}$ & $0.85 \pm 0.49$ & $0.25 \pm 0.03$ & $0.20 \pm 0.07$ & $0.17 \pm 0.05$ & $0.18 \pm 0.05$ \\
\hline $\begin{array}{l}\text { Upstream average } \\
\text { depth }(\mathrm{m})\end{array}$ & $0.29 \pm 0.08$ & $0.36 \pm 0.12$ & $0.50 \pm 0.23$ & $0.52 \pm 0.23$ & $0.18 \pm 0.05$ \\
\hline $\begin{array}{l}\text { Area of the impound- } \\
\text { ment }(\mathrm{Ha})\end{array}$ & 17.14 & 3.08 & 1.96 & 1.77 & 1.03 \\
\hline \multicolumn{6}{|l|}{$\begin{array}{l}\text { Environmental status } \\
\text { Downstream }\end{array}$} \\
\hline Riparian condition & Grass land & $\begin{array}{l}\text { Grass land, } \\
\text { riparian forest }\end{array}$ & $\begin{array}{l}\text { Grass land, } \\
\text { riparian forest }\end{array}$ & $\begin{array}{l}\text { Secondary } \\
\text { forest }\end{array}$ & $\begin{array}{c}\text { Primary and } \\
\text { secondary forest }\end{array}$ \\
\hline Benthic condition & Sand ground & $\begin{array}{l}\text { Rock and } \\
\text { sand ground } \\
\text { macrophyte } \\
\text { presence }\end{array}$ & $\begin{array}{l}\text { Sand ground, } \\
\text { and macrophyte } \\
\text { presence }\end{array}$ & $\begin{array}{l}\text { Sand ground, } \\
\text { and macrophyte } \\
\text { presence }\end{array}$ & $\begin{array}{l}\text { Sand ground, } \\
\text { and macrophyte } \\
\text { presence }\end{array}$ \\
\hline Flow status & lotic water & Lotic water & Lotic water & Lentic water & Lotic water \\
\hline $\begin{array}{l}\text { Upstream } \\
\text { Riparian condition }\end{array}$ & Grass land & $\begin{array}{l}\text { Secondary } \\
\quad \text { forest }\end{array}$ & $\begin{array}{l}\text { Grass land, } \\
\text { riparian forest }\end{array}$ & Grass land & $\begin{array}{l}\text { Grass land, } \\
\text { riparian forest }\end{array}$ \\
\hline Benthic condition & $\begin{array}{l}\text { Rock and } \\
\text { sand ground }\end{array}$ & $\begin{array}{l}\text { Sand ground, } \\
\text { and macrophyte } \\
\text { presence }\end{array}$ & $\begin{array}{l}\text { Sand ground, } \\
\text { and macrophyte } \\
\text { presence }\end{array}$ & $\begin{array}{c}\text { Rock, sand ground, } \\
\text { and macrophyte } \\
\text { presence }\end{array}$ & $\begin{array}{l}\text { Sand ground, } \\
\text { and macrophyte } \\
\text { presence }\end{array}$ \\
\hline Flow status & Lotic water & Lentic water & Lentic water & Lentic water & Lentic water \\
\hline
\end{tabular}

time, high reproductive effort, small body size, low batch fecundity, low investment per offspring and no migratory behavior (15 species) (Table 2). We also performed paired $t$-tests, using species richness per life strategy as response variables. Sampling site position related to the farms, upriver and downriver, were used as factors aiming to test the hypothesis that the impacts of the aquaculture farms were the same for fish species of different life strategies. We found 2 species, Parodon buckleyi and Phenacorhamdia spp., without enough biologic information for a confident classification and an exotic species Oreochromis niloticus that were not included in the analysis.

The distance matrix and the PerMANOVA were performed using the Vegan package (Oksanen et al. 2015), and also the $t$-tests were performed on the $\mathrm{R}$ v.2.14.2 statistical software ( $\mathrm{R}$ Development Core Team 2012, Urbanek et al. 2012).

\section{RESULTS}

A total of 6432 individuals was sampled in the study area, distributed among 4 orders, 20 families and 56 species. Characiformes was the most abundant order with 3987 individuals (61.99\%), followed by Perciformes $(29.60 \%)$, Siluriformes $(4.99 \%)$ and Gymnotiformes (3.42\%). Characiformes was also the most diverse group with 30 species, corresponding to $51 \%$ of the total (Table 2 ).

The highest number of fish was captured at sampling site Pt01, while the lowest occurred at Pt05 (Fig. 2). There was no clear pattern for the number of fish caught between upstream and downstream sites. More fish were caught upstream than downstream at sampling sites Pt01 and Pt02, whereas more fish were caught downstream than upstream at sampling sites Pt03, Pt04 and Pt05 (Fig. 2).

The most frequent species were Serrapinnus aff. microdon, with $15.57 \%(\mathrm{n}=908)$ and Serrapinnus aff. notomelas with $15.29 \%(\mathrm{n}=892)$. Many species were considered rare, with $\leq 5$ individuals collected per species, including Paradon bucleyi, Curimata inornata, Curimata ocellata, Cyphocharax notatus, Hemiodus unimaculatus, Hyphessobrycon agulha, Jupiaba anteroides, Jupiaba cf. apenima, Moenkhausia cotinho, Phenacogaster cf. beni, Roeboides affinis, Ituglanis cf. amazonicus, Corydoras cf. trilineatus, 
Table 2. Fish species, the number of individuals sampled in each location (Pt01-05, see Fig. 1) and their life history strategy (LHS). Up: upstream; Dn: down-stream of the fish farm plant

\begin{tabular}{|c|c|c|c|c|c|c|c|c|c|c|c|}
\hline \multirow[t]{2}{*}{ Taxon } & \multicolumn{2}{|c|}{ Pt01 } & \multicolumn{2}{|c|}{ Pt02 } & \multicolumn{2}{|c|}{ Pt03 } & \multicolumn{2}{|c|}{ Pt04 } & \multicolumn{2}{|c|}{ Pt05 } & \multirow[t]{2}{*}{ LHS } \\
\hline & Up & Dn & Up & Dn & Up & Dn & Up & Dn & Up & Dn & \\
\hline \multicolumn{12}{|l|}{ CHARACIFORMES } \\
\hline \multicolumn{12}{|l|}{ Parodontidae } \\
\hline Parodon buckleyi Boulenger, 1887 & & & & 2 & & 1 & & & & & Unknown \\
\hline \multicolumn{12}{|l|}{ Curimatidae } \\
\hline Curimata inornata Vari, 1989 & & & & & & & & 1 & & & Periodic \\
\hline Curimata ocellata Eigenmann \& Eigenmann, 1889 & & & & & & & & 2 & & & Periodic \\
\hline Curimatella dorsalis (Eigenmann \& Eigenmann, 1889) & 35 & 34 & & & & & & 20 & 6 & 72 & Periodic \\
\hline Cyphocharax notatus (Steindachner, 1908) & & & & & & & & & & 1 & Periodic \\
\hline Steindachnerina fasciata (Vari \& Géry, 1985) & 112 & 220 & 25 & 34 & 19 & 101 & & 5 & & 8 & Periodic \\
\hline \multicolumn{12}{|l|}{ Prochilodontidae } \\
\hline Prochilodus nigricans Spix \& Agassiz, 1829 & & 1 & & 7 & & 3 & & 10 & & 7 & Periodic \\
\hline \multicolumn{12}{|l|}{ Anostomidae } \\
\hline Leporinus friderici (Bloch, 1794) & & 39 & & 4 & & 1 & & 3 & & 1 & Periodic \\
\hline \multicolumn{12}{|l|}{ Crenuchidae } \\
\hline Characidium aff. zebra Eigenmann, 1909 & 7 & 1 & & & & & & & & & Opportunistic \\
\hline \multicolumn{12}{|l|}{ Hemiodontidae } \\
\hline Hemiodus unimaculatus (Block, 1794) & & & & & & & & 1 & & & Periodic \\
\hline \multicolumn{12}{|l|}{ Characidae } \\
\hline Astyanax aff. bimaculatus (Linnaeus, 1758) & 4 & 68 & 11 & 54 & 110 & 119 & & 38 & & 3 & Periodic \\
\hline Astyanax cf. maximus (Steindachner, 1876) & & 1 & & 27 & & & & 3 & 1 & 11 & Periodic \\
\hline Brachychalcinus copei (Steindachner, 1882) & & & 1 & 17 & & 1 & & 4 & & & Opportunistic \\
\hline Hyphessobrycon agulha Fowler, 1913 & & & & & & & & & 1 & & Periodic \\
\hline Jupiaba anteroides (Géry, 1965) & & & & & & & & & & 5 & Opportunistic \\
\hline Jupiaba cf. apenima Zanata, 1997 & & & & & & & & & & 5 & Opportunistic \\
\hline Knodus cf. heteresthes (Eigenmann, 1908) & & 1 & 14 & 25 & 10 & 46 & & & & & Opportunistic \\
\hline Moenkhausia cf. pankilopteryx Bertaco \& Lucinda, 2006 & & & 1 & 19 & & & & & & 4 & Opportunistic \\
\hline Moenkhausia cotinho Eigenmann, 1908 & & & & & & & & 5 & & & Periodic \\
\hline Moenkhausia oligolepis (Günther, 1864) & & 1 & 26 & 11 & 2 & 2 & & & 3 & 19 & Opportunistic \\
\hline Phenacogaster cf. beni Eigenmann, 1911 & & & & & 1 & & & & & & Opportunistic \\
\hline Poptella compressa (Günther, 1864) & & & & & & & & 9 & & & Opportunistic \\
\hline Roeboides affinis (Günther, 1868) & & & & & & & & & & 2 & Opportunistic \\
\hline Serrapinnus aff. microdon (Eigenmann, 1915) & 181 & 296 & 161 & 9 & 64 & 138 & & 15 & 3 & 41 & Opportunistic \\
\hline Serrapinnus aff. notomelas (Eigenmann, 1915) & 217 & 54 & 185 & 31 & 14 & 103 & & 172 & 108 & 8 & Opportunistic \\
\hline \multicolumn{12}{|l|}{ Serrasalmidae } \\
\hline Myloplus asterias (Müller \& Troschel, 1844) & & & & 7 & & & & 1 & & & Equilibrium \\
\hline Serrasalmus altispinis Merckx, Jégu \& Santos, 2000 & 40 & 65 & & & & & & 8 & & & Opportunistic \\
\hline \multicolumn{12}{|l|}{ Iguanodectidae } \\
\hline Bryconops cf. giacopinii (Fernández-Yépez, 1950) & 1 & 11 & 2 & 29 & & 20 & & 67 & 5 & 15 & Periodic \\
\hline Acestrorhynchidae & & & & & & & & & & & \\
\hline Acestrorhynchus falcatus (Bloch, 1794) & & & & & & & & & & 6 & Periodic \\
\hline Erythrinidae & & & & & & & & & & & \\
\hline Hoplias malabaricus (Bloch, 1794) & 27 & 4 & 20 & 2 & 16 & 4 & 6 & 9 & 4 & 1 & Opportunistic \\
\hline SILURIFORMES & & & & & & & & & & & \\
\hline Trichomycteridae & & & & & & & & & & & \\
\hline Ituglanis cf. amazonicus (Steindachner, 1882) & & & & & & & & & & 1 & Equilibrium \\
\hline Callichthyidae & & & & & & & & & & & \\
\hline Callichthys callichthys (Linnaeus, 1758) & & & & 2 & & 9 & & 16 & & & Equilibrium \\
\hline Corydoras aeneus (Gill, 1858) & & & 5 & & 35 & & & & & & Equilibrium \\
\hline Corydoras cf. trilineatus Cope, 1872 & & & & 1 & & & & & & & Equilibrium \\
\hline Hoplosternun littorale (Hancock, 1828) & & 3 & 14 & 1 & & 10 & & & & & Equilibrium \\
\hline
\end{tabular}


Table 2 (continued)

\begin{tabular}{|c|c|c|c|c|c|c|c|c|c|c|c|}
\hline \multirow{2}{*}{ Taxon } & \multicolumn{2}{|c|}{ Pt01 } & \multicolumn{2}{|c|}{ Pt02 } & \multicolumn{2}{|c|}{ Pt03 } & \multicolumn{2}{|c|}{ Pt04 } & \multicolumn{2}{|c|}{ Pt05 } & \multirow{2}{*}{ LHS } \\
\hline & Up & Dn & Up & Dn & Up & Dn & Up & Dn & Up & $\mathrm{Dn}$ & \\
\hline \multicolumn{12}{|l|}{ Loricariidae } \\
\hline Ancistrus cf. dubius Eigenmann \& Eigenmann, 1889 & 1 & 3 & 9 & 11 & & 6 & & 3 & & 1 & Equilibrium \\
\hline Farlowella oxyrrhyncha (Kner, 1853) & & & 1 & 6 & & & 1 & 2 & & & Equilibrium \\
\hline Hypostomus pyrineusi (Miranda Ribeiro, 1920) & 1 & 5 & 6 & 11 & 3 & 16 & & 30 & & 1 & Equilibrium \\
\hline Hypostomus spp. & & & & 2 & & & & 3 & & & Equilibrium \\
\hline Pterygoplichthys lituratus (Kner, 1854) & & 2 & & & & & & 1 & 2 & 2 & Equilibrium \\
\hline Rineloricaria lanceolata (Günther, 1868) & & & & 7 & 1 & 13 & & 1 & & & Equilibrium \\
\hline Rineloricaria spp. 'Juruema' & & 20 & & 1 & 2 & 14 & & 6 & & & Equilibrium \\
\hline \multicolumn{12}{|l|}{ Heptapteridae } \\
\hline Phenacorhamdia spp. & & & & & & & & & & 1 & Unknown \\
\hline Pimelodella serrata Eigenmann, 1917 & & & 7 & 6 & & 3 & & & & & Equilibrium \\
\hline Rhamdia quelen (Quoy \& Gaimard, 1824) & & 3 & & & 8 & 1 & & & & & Equilibrium \\
\hline \multicolumn{12}{|l|}{ Auchenipteridae } \\
\hline Trachelyopterus galeatus (Linnaeus, 1766) & & & & & & & & 1 & & & Equilibrium \\
\hline \multicolumn{12}{|l|}{ GYMNOTIFORMES } \\
\hline \multicolumn{12}{|l|}{ Gymnotidae } \\
\hline Gymnotus carapo Linnaeus, 1758 & & 21 & 10 & 4 & 33 & 28 & 9 & 4 & 2 & 4 & Equilibrium \\
\hline \multicolumn{12}{|l|}{ Sternopygidae } \\
\hline Eigenmannia sp. nov. & & 17 & & 9 & 5 & 15 & & 9 & & 8 & Opportunistic \\
\hline Sternopygus macrurus (Bloch \& Schneider, 1801) & 6 & 2 & & 5 & 6 & 14 & & 3 & & 2 & Equilibrium \\
\hline \multicolumn{12}{|l|}{ Apteronotidae } \\
\hline Apteronotus albifrons (Linnaeus, 1766) & & & & 1 & & & & 1 & & 2 & Equilibrium \\
\hline \multicolumn{12}{|l|}{ PERCIFORMES } \\
\hline \multicolumn{12}{|l|}{ Cichlidae } \\
\hline Aequidens tetramerus (Heckel, 1840) & 113 & 72 & 118 & 81 & 151 & 104 & 86 & 6 & 22 & 14 & Equilibrium \\
\hline Cichla monoculus Agassiz, 1831 & 3 & & & 3 & & & & 9 & & & Equilibrium \\
\hline Crenicichla lepidota Heckel, 1840 & 6 & 23 & 4 & 2 & 8 & 2 & & 6 & 4 & 2 & Equilibrium \\
\hline Crenicichla spp. & & & & 5 & 27 & 14 & & 12 & & 8 & Equilibrium \\
\hline Oreochromis niloticus (Linnaeus, 1758) & & & & & 8 & 1 & & 1 & & & Not classified \\
\hline Satanoperca jurupari (Heckel, 1840) & 282 & 228 & 1 & 13 & 23 & 47 & & 48 & 64 & 33 & Equilibrium \\
\hline
\end{tabular}

Hypostomus spp., Phenacorhamdia spp., Trachelyopterus galeatus, and Apteronotus albifrons. In addition, 10 individuals of the exotic species Oreochromis niloticus were collected.

A clearer pattern was observed for ecological measures. All sampling sites showed lower richness $(S)$ at locations upstream of the dams (Table 3, Fig. 3). Shannon's $\left(H^{\prime}\right)$ index was also lower in the upstream areas, indicating that overall diversity was higher downstream than upstream. Evenness $(E)$ also showed more equitability downstream than upstream, with the exception of Pt03, which exhibited similar values of evenness for both areas. With exception of Pt01, the Berger-Parker $(D)$ index shows that species dominance was highest in upstream locations (Table 3).

The test for homogeneity of multivariate dispersions did not reveal negative eigenvalues, indicating that there is multivariate homogeneity between groups. PERMANOVA rejected the null hypothesis for similarity between upstream and downstream locations at each site (Pseudo- $F=1.373$; $\mathrm{df}=1,16 ; \mathrm{p}=$ 0.036), indicating that species composition was not the same between the sampling points upstream and downstream of the aquaculture dams. We detected no differences between seasons of the hydrologic cycle (Pseudo- $F=0.312 ; \mathrm{df}=1,16 ; \mathrm{p}=0.909$ ) and no interaction effects (Pseudo- $F=0.335$; df $=1,16 ; \mathrm{p}=$ $0.887)$. However, water quality parameters did not show significant differences between upstream and downstream locations when submitted to the paired $t$-test presenting $\mathrm{p}>0.05$ (Table 4).

The paired $t$-test performed using species richness clustered by life history strategies showed significant differences between up- and downstream locations for equilibrium $(t=-3.428, \mathrm{df}=4, \mathrm{p}=0.026)$ and periodic $(t=-2.954, \mathrm{df}=4, \mathrm{p}=0.042)$, with species rich- 


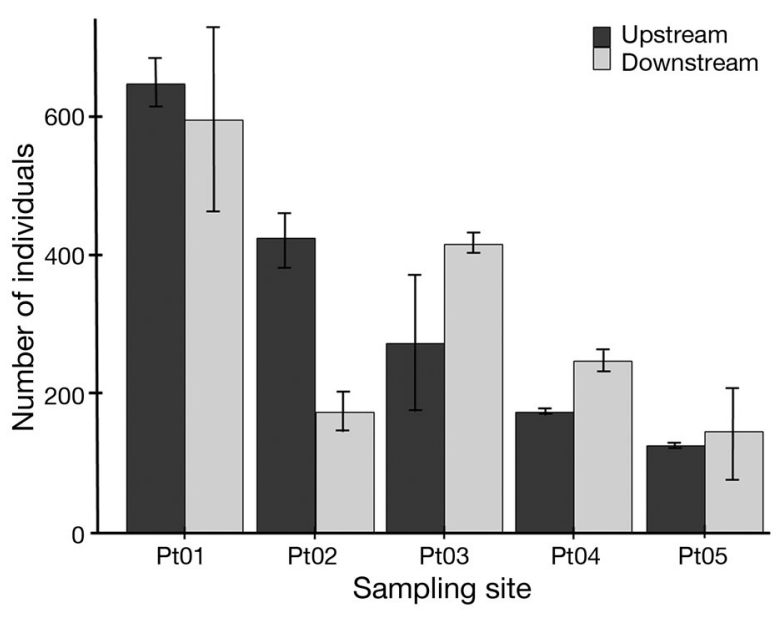

Fig. 2. Number of fish (mean $\pm \mathrm{SD}$ ) caught both upstream and downstream of aquaculture dams at the sampling sites

ness higher at the sampling sites located down-river for both fish groups. No difference was observed for opportunistic species $(t=-1.856, \mathrm{df}=4, \mathrm{p}=0.137)$.

\section{DISCUSSION}

Pelicice et al. (2015) hypothesized that reservoirs of large dams act as ecologic barriers for downstream fish movements due to the presence of a gradient of hydraulic and limnological features. However,

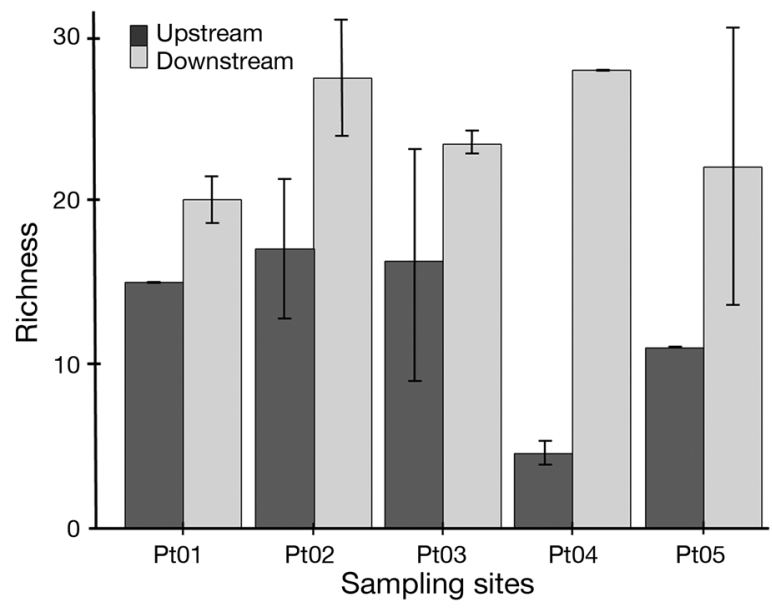

Fig. 3. Fish species richness distribution (mean \pm SD) both upstream and downstream of aquaculture dams at the sampling sites

whether they are small or large, dams primarily impede upstream fish movements, especially if they do not have effective accommodations for fish to bypass these barriers. Allowing for fish to bypass dam barriers is likely to be easier in small streams where the slope is shallower.

Our study focused on small dams and detected similar effects to the ones reported for larger dams. Indeed, most of the studies on river regulation at large hydroelectric developments report an alteration in species richness and diversity, and consequently in

Table 3. Estimates of fish richness $(S)$, abundance $(\mathrm{N})$, diversity index $\left(H^{\prime}\right)$, dominance index $(D)$, and evenness $(E)$ from upstream (up) and downstream (down) of each sampling site (Pt01-05, see Fig. 1)

\begin{tabular}{|c|c|c|c|c|c|c|c|c|c|c|}
\hline \multirow{2}{*}{$\begin{array}{l}\text { Fish assem- } \\
\text { blage attribute }\end{array}$} & \multicolumn{2}{|c|}{ Pt01 } & \multicolumn{2}{|c|}{ Pt02 } & \multicolumn{2}{|c|}{ Pt03 } & \multicolumn{2}{|c|}{ Pt04 } & \multicolumn{2}{|c|}{ Pt05 } \\
\hline & Up & Down & Up & Down & Up & Down & Up & Down & Up & Down \\
\hline$S$ & 18 & 25 & 20 & 34 & 21 & 28 & 5 & 36 & 15 & 29 \\
\hline $\mathrm{N}$ & 1,299 & 1,194 & 841 & 349 & 546 & 836 & 340 & 497 & 248 & 282 \\
\hline$H^{\prime}$ & 1.981 & 2.269 & 2.048 & 3.100 & 2.316 & 2.562 & 0.574 & 2.532 & 1.711 & 2.617 \\
\hline$D$ & 0.2402 & 0.2479 & 0.2592 & 0.0974 & 0.2766 & 0.1651 & 0.8412 & 0.3461 & 0.4355 & 0.2553 \\
\hline$E$ & 0.6853 & 0.7050 & 0.6835 & 0.8792 & 0.7606 & 0.7689 & 0.3572 & 0.7067 & 0.6317 & 0.7772 \\
\hline
\end{tabular}

Table 4 . Water quality parameters (mean \pm SD) measured at sampling sites (Pt01-05, see Fig. 1), during the years 2014 and 2015 considering 2 seasons including day and night samples from upstream and downstream locations $(n=8)$. EC: electrical conductivity

\begin{tabular}{|llcccrc|}
\hline \multirow{2}{*}{ Location } & Limnological parameter & Pt01 & Pt02 & Pt03 & Pt04 & Pt05 \\
\hline Upstream & Dissolved oxygen $\left(\mathrm{mg} \mathrm{l}^{-1}\right)$ & $3.65 \pm 1.32$ & $2.07 \pm 0.79$ & $3.47 \pm 1.23$ & $4.85 \pm 0.61$ & $4.67 \pm 1.45$ \\
& pH & $6.02 \pm 0.87$ & $6.37 \pm 0.66$ & $5.97 \pm 1.18$ & $5.47 \pm 0.83$ & $6.02 \pm 0.44$ \\
& Temperature $\left({ }^{\circ} \mathrm{C}\right)$ & $27.50 \pm 1.58$ & $26.67 \pm 2.49$ & $25.31 \pm 0.76$ & $24.85 \pm 0.61$ & $26.42 \pm 3.46$ \\
& EC $\left(\mu \mathrm{S} \mathrm{cm}^{-1}\right)$ & $55.60 \pm 12.97$ & $97.00 \pm 17.32$ & $103.07 \pm 14.85$ & $60.17 \pm 15.36$ & $13.50 \pm 2.69$ \\
Downstream & Dissolved oxygen $\left(\mathrm{mg} \mathrm{l}^{-1}\right)$ & $4.60 \pm 2.99$ & $3.65 \pm 2.08$ & $4.75 \pm 0.66$ & $5.72 \pm 2.19$ & $5.31 \pm 0.78$ \\
& pH & $6.17 \pm 1.32$ & $6.64 \pm 0.79$ & $6.26 \pm 1.08$ & $5.73 \pm 1.31$ & $6.00 \pm 0.17$ \\
& Temperature $\left({ }^{\circ} \mathrm{C}\right)$ & $28.30 \pm 0.94$ & $26.92 \pm 0.89$ & $26.30 \pm 1.14$ & $27.72 \pm 2.19$ & $27.98 \pm 1.80$ \\
& EC $\left(\mu \mathrm{Sm}^{-1}\right)$ & $59.52 \pm 9.73$ & $119.80 \pm 24.80$ & $112.20 \pm 14.07$ & $51.15 \pm 32.62$ & $13.60 \pm 2.77$ \\
& & & & & & \\
\hline
\end{tabular}


assemblage composition (Pusey et al. 1995, ReyesGavilán et al. 1996, Gehrke et al. 2002). However, we hypothesized that there are different causal mechanisms. We believe that the short distance between sampling points located up- and downstream of these small dams, not large enough to be linked to environmental/landscape differences between these sampling points, which supports the hypothesis that a physical barrier is the main factor to explain the observed differences. It appears that dams in smaller streams block upstream movements of resident fish, probably associated with reproductive and feeding strategies, thereby reducing fish diversity in the headwater areas. The results here suggest that dams separated these fish assemblages, leading to the isolation of certain fish groups in upstream areas. In comparison, fish in downstream areas have free movement to access the Machado River and its other tributaries. The fish remaining upstream effectively become trapped in a smaller area and face increasing competition and predation pressures that may eliminate some species, especially if they are rare or require downstream access as part of their life cycles.

The magnitude of the dams' effect is associated with fish life strategy. Some of the most abundant periodic species, such as Steindachnerina fasciata, Astyanax aff. bimaculatus and Bryconops cf. giacopinii, showed reduced abundance in upstream areas and were dominant in downstream areas. In the sense of Winemiller \& Rose (1992), this group includes mostly migratory species and therefore should be particularly sensitive to the connectivity loss resulting from physical barriers imposed by aquaculture farms in the river channel.

Equilibrium species includes most groups belonging to Siluriformes, Gymnotiformes and Perciformes. We hypothesized that the unclear pattern observed for equilibrium species could be explained by the different level of demographic compensation via dispersal exhibited by these species (Rose et al. 2001). Two equilibrium species were very abundant up- and downstream-Aequidens tetramerus and Satanoperca jurupari-without a clear pattern of dominance associated with the position related to the aquaculture farm. Both are small-size fish and exhibit life history traits that could permit survival even with the loss of connectivity.

Finally, opportunistic species showed no effect of dams' presence. The high abundance of sedentary and opportunistic species in upstream areas could be associated with the new environment, predominantly lentic, caused by the stream impoundment (Loureiro \& Hahn 1996, Hoshino et al. 2016, Oliveira et al.
2016). These slow-moving environments are favorable for these species to complete their life cycle because they do not rely on seasonal variations in water level to reproduce (Graça \& Pavanelli 2007, Queiroz et al. 2013, Zuanon et al. 2015). They are predominantly small-sized species (e.g. small pelagic forage fish species), which exhibit high abundance and fecundity (Hahn \& Fugi 2007).

A critical threat associated with upstream isolation is that it could end up causing localized extinction of rare and endemic species, but in our study, we were not able to sample these sites before the streams were dammed. Comparisons with such areas as controls would be important to determine if species suffer declines or extinctions as a result of impoundments of fish farms. Headwater areas of Amazonian streams, regionally called igarapés, host several rare and endemic species adapted to these environments, as small streams (Mendonça et al. 2005, EspíritoSanto et al. 2009). One of the consequences of permanent flooding due to dam impoundment is the elimination of temporary pools adjacent to the stream channels that form after heavy rains, which are known to be important to the stability of fish assemblages in headwater areas of Amazonian streams (Espírito-Santo \& Zuanon 2016). However, these newly flooded environments could create further niches for species that require more slow-moving habitats for feeding or reproduction.

A final consideration related to impacts caused by fish farming activity of tambaqui in the Machado River basin is the presence of an introduced invasive species, the tilapia Oreochromis niloticus, which is another common aquaculture species (Zambrano et al. 2006). Tilapia has been spreading invasively throughout Brazilian waterways due to escapes from aquaculture pens, causing competition with native species for space and food. Furthermore, tilapia has been disseminating parasites and diseases among native fish (Fernandes et al. 2008, Miranda et al. 2010, Miranda 2012), putting in peril native fish communities (Agostinho et al. 1999, Dias 2016).

We conclude that there are 2 important agendas to follow: (1) create and test more natural fish passage systems (FAO/DVWK 2002) as alternatives to fish ladders to reduce the impact of impoundments on aquatic biodiversity; (2) prevent introductions of nonnative fish to the Amazon Basin, substituting native species to tilapia in fish farming. Given the high rate at which anthropogenic changes are occurring in the Amazonian environment (Hurd et al. 2016), the urgency for research aimed at preserving the diversity of these fish communities could hardly be greater. 
Acknowledgements. Many thanks to Alex Junior Vervloet Braz for his help in the field with fish samples, also for his help during the fish identification. We also acknowledge the anonymous reviewers for their helpful comments on the manuscript; and the National Council of Technological and Scientific Development (CNPq) and Rondônia Federal University for funding and logistical support of this study.

\section{LITERATURE CITED}

Agostinho AA, Gomes LC, Suzuki HI, Júlio JRHF (1999) Riscos da implantação de cultivos de espécies exóticas em tanques-redes em reservatórios do Rio Iguaçu. Cadernos da Biodiversidade 2:1-9

Agostinho AA, Gomes LC, Pelicice FM (2007) Ecologia e manejo de recursos pesqueiros em reservatórios do Brasil. EDUEM, Maringá

Agostinho AA, Pelicice FM, Gomes LC (2008) Dams and the fish fauna of the Neotropical region: impacts and management related to diversity and fisheries. Braz J Biol 68: 1119-1132

Agostinho AA, Agostinho CS, Pelicice FM, Marquis EE (2012) Fish ladders: safe fish passage or hotspot for predation? Neotrop Ichthyol 10:687-696

Albanez JR, Albanez ACMP (2000) Legislação ambiental aplicada à piscicultura. Ufla/Faepe, Lavras

Anderson M (2001) A new method for non-parametric multivariate analysis of variance. Austral Ecol 26:32-46

Arantes CC, Winemiller KO, Petrere M, Castello L, Hess LL, Freitas CEC (2017) Relationships between forest cover and fish diversity in the Amazon River floodplain. J Appl Ecol (in press) doi:10.1111/1365-2664.12967

Barletta M, Jaureguizar AJ, Baigun C, Fontoura NF and others (2010) Fish and aquatic habitat conservation in South America: a continental overview with emphasis on Neotropical systems. J Fish Biol 76:2118-2176

Buttigieg PL, Ramette A (2014) A guide to statistical analysis in microbial ecology: a community-focused, living review of multivariate data analysis. FEMS Microbiol Ecol 90:543-550

Carvalho RA, Tejerina-Garro FL (2015) The influence of environmental variables on the functional structure of headwater stream fish assemblages: a study of two tropical basins in Central Brazil. Neotrop Ichthyol 13: 349-360

Castello L, Macedo MN (2016) Large-scale degradation of Amazonian freshwater ecosystems. Glob Change Biol 22:990-1007

* Cox-Fernandes CC (1997) Lateral migrations of fishes in Amazon floodplains. Ecol Freshwat Fish 6:36-44

Dias MT (2016) Invasão da tilápia-do-nilo no Estado do Amapá: uma ameaça aos peixes nativos da Bacia Igarapé Fortaleza. Documentos 92, Embrapa Amapá, Macapá

* Espírito-Santo HMV, Zuanon J (2017) Temporary pools provide stability to fish assemblages in Amazon headwater streams. Ecol Freshwat Fish 26:475-483

Espírito-Santo HMV, Magnusson WE, Zuanon J, Mendonça FP, Landeiro VL (2009) Seasonal variation in the composition of fish assemblages in small Amazonian forest streams: evidence for predictable changes. Freshw Biol 54:536-548

FAO/DVWK (2002) Fish passes-design, dimensions and monitoring. FAO, Rome

Fernandes R, Gomes LC, Agostinho AA (2008) Pesque- pague: negócio ou fonte de dispersão de espécies exóticas? Acta Sci Biol Sci 25:115-120

*Finer M, Jenkins CN (2012) Proliferation of hydroelectric dams in the Andean Amazon and its implications for Andes-Amazon connectivity. PLOS ONE 7:e35126

Freitas CEC, Garcez RCS (2004) Fish communities of natural canals between floodplain lakes and Solimões-Amazonas River (Amazon-Brazil). Acta Limnol Bras 16:273-280

*Gehrke PC, Gilligan DM, Barwick M (2002) Changes in fish communities of the Shoalhaven River 20 years after construction of Tallowa Dam, Australia. River Res Appl 18: 265-286

Girard P (2002) Efeito cumulativo das barragens no Pantanal. Instituto Centro Vida, Campo Grande

Graça WJ, Pavanelli CS (2007) Peixes da planície de inundação do alto rio Paraná e áreas adjacentes. Eduem, Maringá

*Hahn NS, Fugi R (2007) Alimentação de peixes em reservatórios brasileiros: alterações e conseqüências nos estágios iniciais do represamento. Revista Oecologia Brasiliensis 4:469-480

* Hoshino MDFG, Neves LR, Tavares-Dias M (2016) Comunidades parasitárias dos peixes predadores, Acestrorhynchus falcatus e Acestrorhynchus falcirostris, que vivem em simpatria na Amazônia brasileira. Rev Bras Parasitol Vet 25:207-216

*Hurd LE, Sousa RGC, Siqueira-Souza FK, Cooper GJ, Kahn JR, Freitas CEC (2016) Amazon floodplain fish communities: habitat connectivity and conservation in a rapidly deteriorating environment. Biol Conserv 195:118-127

Kahn JR, Freitas CEC, Petrere M (2014) False shades of green: the case of Brazilian Amazonian hydropower. Energies 7:6063-6082

*Lima LB, Oliveira FJM, Giacomini HC, Lima-Junior AP (2016) Expansion of aquaculture parks and the increasing risk of non-native species invasions in Brazil. Rev Aquacult (in press), doi:10.1111/raq.12150

Loureiro VE, Hahn NS (1996) Dieta e atividade alimentar da traíra, Hoplias malabaricus (Bloch, 1794) (Osteichthyes, Erythrinidae), nos primeiros anos de formação do Reservatório de Segredo - PR. Acta Limnol Bras 8:195-205

Maltchik L, Medeiros ESF (2006) Diversidade, estabilidade e atividade reprodutiva de peixes em uma poça fluvial permanente no leito de um riacho efêmero, riacho Avelós, Paraíba, Brasil. Revista de Biologia e Ciências da Terra 1:20-28

Mendonça FP, Magnusson WE, Zuanon J (2005) Relationships between habitat characteristics and fish assemblages in small streams of Central Amazonia. Copeia $750-763$

Miranda JC (2012) Ameaças aos peixes de riachos da Mata Atlântica. Natureza On Line (Espírito Santo) 10:136-139

Miranda JC, Mazzoni R, Silva CEA (2010) Ocorrência da tilápia do Nilo Oreochromis niloticus Linnaeus, 1758 na microbacia do rio Mato Grosso, Saquarema, Rio de Janeiro. Revista de Saúde e Biologia 5:47-50

MPA (Ministério da Pesca e Aquicultura) (2010) Produção pesqueira e aquícola, 2010. Estatística 2008 e 2009. MPA, Brasilia. www.icmbio.gov.br/cepsul/images/stories/ biblioteca/download/estatistica/est_2008_2009_nac_pesca. pdf (accessed 29/01/2018)

Neto RM, Nocko HR, Ostrensky A (2015) Environmental characterization and impacts of fish farming in the cascade reservoirs of the Paranapanema River, Brazil. Aquacult Environ Interact 6:255-272 
Oksanen JF, Blanchet G, Kindt R, Legendre P and others (2015) Vegan: community ecology package version 2.2-1:17-19. www.pelagicos.net/MARS6910_spring2015/ manuals/R_vegan.pdf

Oliveira JF, Moraes-Segundo AL, Novaes JL, Costa RS, França JS, Peretti D (2016) Trophic structure of the ichthyofauna in a Brazilian semiarid reservoir. Iheringia Ser Zool 106:1-9

Ostrensky A, Borghetti JR, Soto D (2008) Aquicultura no Brasil: o desafio é crescer. Secretaria Especial de Aquicultura e Pesca da Presidência da República, Brasília

Pelicice, FM, Agostinho AA (2008) Fish-passage facilities as ecological traps in large neotropical rivers. Conserv Biol 22:180-188

Pelicice FM, Pompeu PS, Agostinho AA (2015) Large reservoirs as ecological barriers to downstream movements of Neotropical migratory fish. Fish Fish 16:697-715

Pereira LS, Agostinho AA, Delariva RL (2016) Effects of river damming in Neotropical piscivorous and omnivorous fish: feeding, body condition and abundances. Neotrop Ichthyol 14:e150044

* Pusey BJ, Arthington AH, Read MG (1995) Species richness and spatial variation in fish assemblage structure in two rivers of the Wet Tropics of northern Queensland, Australia. Environ Biol Fishes 42:181-199

Queiroz LJ, Torrente-Vilara G, Ohara WM, Pires THS, Zuanon J, Doria CRC (2013) Peixes do Rio Madeira. Dialeto Latin American Documentary, São Paulo

R Development Core Team (2012) R: a language and environmental for statistical computing. R Foundation for Statistical Computing, Vienna. www.r-project.org

Reyes-Gavilán FG, Garrido R, Nicieza AG, Toledo MM, Braña F (1996) Fish community variation along physical gradients in short streams of northern Spain and the disruptive effect of dams. Hydrobiologia 321:155-163

Editorial responsibility: Pablo Sánchez Jerez, Alicante, Spain
Ribeiro MCLDB, Petrere M, Juras AA (1995) Ecological integrity and fisheries ecology of the Araguaia-Tocantins River Basin, Brazil. Regul Rivers Res Manage 11:325-350

Rondônia (2015) Plano de desenvolvimento estadual sustentável de Rondônia, 2015-2030. Con\&Sea, Porto Velho

Röpke CP, Amadio S, Zuanon J, Ferreira EFG, de Deus CP, Pires THS, Winemiller KO (2017) Simultaneous abrupt shifts in hydrology and fish assemblages structure in a floodplain lake in the Central Amazon. Sci Rep 7:40170

Rose KA, Cowan JH Jr, Winemiller KO, Myers RA, Hilborn $\mathrm{R}$ (2001) Compensatory density dependence in fish populations: importance, controversy, understanding and prognosis. Fish Fish 2:293-327

* Sousa RGC, Freitas CEC (2008) The influence of flood pulse on fish communities of floodplain canals in the Middle Solimões River, Brazil. Neotrop Ichthyol 6:249-255

*Winemiller KO (2005) Life history strategies, population regulation, and implications for fisheries management. Can J Fish Aquat Sci 62:872-885

* Winemiller KO, Rose KA (1992) Patterns of life-history diversification in North American fishes: implications for population regulation. Can J Fish Aquat Sci 49: 2196-2218

*Winemiller KO, Mcintyre PB, Castello L, Fluet-Chouinard E and others (2016) Balancing hydropower and biodiversity in the Amazon, Congo, and Mekong. Science 351: 128-129

Zambrano L, Martínez-Meyer H, Menezes N, Peterson AT (2006) Invasive potential of common carp (Cyprinus carpio) and Nile tilapia (Oreochromis niloticus) in American freshwater systems. Can J Fish Aquat Sci 63: 1903-1910

Zuanon J, Mendonça FP, Espírito Santo HMV, Dias MS, Galuch AV, Akama A (2015) Guia de peixes da Reserva Adolpho. Editora INPA, Manaus

Submitted: April 26, 2017; Accepted: December 4, 2017 Proofs received from author(s): January 30, 2018 